\title{
PERCEPÇÕES E SIGNIFICADOS SOBRE O ATENDIMENTO DE CRIANÇAS EM SITUAÇÃO DE EMERGÊNCIA
}

\section{PERCEPTIONS AND MEANINGS ABOUT THE CARE OF CHILDREN IN EMERGENCY}

\section{PERCEPCIONES Y SIGNIFICADOS SOBRE EL CUIDADO DE LOS HIJOS EN SITUACÓN DE EMERGENCIA}

Geovanna de Oliveira Barreto ${ }^{1}$, Elen Stefani Lima Silva ${ }^{2}$, Nylze Helena Guillarducci Rocha ${ }^{3}$, Luana Barbosa Zago ${ }^{4}$, Jesislei Bonolo Amaral Rocha ${ }^{5}$, Divanice Contim ${ }^{6}$

Como citar esse artigo: Barreto GO, Silva ESL, Rocha NHG, Zago LB, Rocha JBA, Contim D. Percepções e significados sobre o atendimento de crianças em situação de emergência. Rev Enferm Atenção Saúde [Online]. 2021 [acesso em:__];10(1):e202107. doi: https://doi.org/ 10.18554/reas.v10i1.4175

\section{RESUMO}

Objetivo: Este estudo pretende discutir e analisar as percepções de uma equipe de enfermagem de um Pronto Socorro Infantil, sobre o atendimento às crianças em situação emergencial. Método: Estudo de abordagem qualitativa e descritiva. O cenário para coleta de dados foi uma Unidade de Pronto Socorro Infantil de um hospital de ensino, localizado no estado de Minas Gerais, com membros da equipe de enfermagem entrevistados por meio de um questionário semiestruturado. Os dados foram analisados utilizando-se a técnica de análise de conteúdo. Resultados: Participaram do estudo 10 profissionais de enfermagem, todos do sexo feminino, sendo duas enfermeiras e oito técnicas de enfermagem. Foram identificadas percepções e significados sobre o conhecimento, a responsabilidade, as dificuldades, as necessidades de protocolos e as formas de aprendizagem do atendimento. Conclusão: Constatou-se que o conhecimento, prática, responsabilidade, habilidade e comunicação são fatores que possibilitam uma assistência segura à criança e seus familiares em situação de urgência e emergência.

Descritores: Enfermagem; Criança; Equipe de enfermagem; Medicina de Emergência Pediátrica.

\footnotetext{
1 Enfermeira, especialista em Saúde da Criança e do Adolescente, UFTM-MG. E-mail: geovanna_obarreto@hotmail.com

2 Graduanda em Enfermagem pela UFTM-MG. http://orcid.org/0000-0003-3910-2963, e-mail: elen.setafany@gmail.com

3 Enfermeira, especialista em Enfermagem Neonatal, Mestranda em Atenção à Saúde - UFTM-MG. http://orcid.org/0000-0002-6615-1667, e-mail: nylze@ hotmail.com

${ }^{4}$ Enfermeira, Responsável Técnica do Setor de Pronto Socorro, HC UFTM-MG.

5 Enfermeira, Professora Doutora, UFTM-MG. http://orcid.org/0000-0002-0591-7972, e-mail: jesisleiamaralrocha@gmail.com

6 Enfermeira. Professora Doutora, UFTM-MG. http://orcid.org/0000-0001-5213-1465, e-mail: d.contim@uol.com.br
} 


\begin{abstract}
Objective: This study aims to discuss and analyze how perceptions of a nursing team in an Infant Emergency Room, regarding the care of children in emergency. Method: Study with a qualitative and descriptive approach. The scenario for data collection was a Children's Emergency Unit in a teaching hospital, located in the state of Minas Gerais, with members of the nursing team interviewed using a semi-structured questionnaire. The data were compensated using the content analysis technique. Results: 10 nursing professionals participated in the study, all female, two nurses and eight nursing technicians. Perceptions and meanings about knowledge, responsibility, difficulties, protocols needs and forms of service learning were identified. Conclusion: It was found that knowledge, practice, responsibility, skill and communication are factors that enable safe care for children and their families in situations of urgency and emergency.
\end{abstract}

Descriptors: Nursing; Child; Nursing team; Pediatric Emergency Medicine.

\title{
RESUMEN
}

Objetivo: Este estudio tiene como objetivo discutir y analizar cómo las percepciones de un equipo de enfermería en una Sala de Emergencias Infantiles, respecto al cuidado de los niños en situaciones de emergencia. Método: Estudio con enfoque cualitativo y descriptivo. El escenario para la recolección de datos fue una Unidad de Emergencia Infantil en un hospital universitario, ubicado en el estado de Minas Gerais, con miembros del equipo de enfermería entrevistados mediante un cuestionario semiestructurado. Los datos se compensaron mediante la técnica de análisis de contenido. Resultados: participaron del estudio 10 profesionales de enfermería, todas mujeres, dos enfermeras y ocho técnicos de enfermería. Se identificaron percepciones y significados sobre conocimientos, responsabilidad, dificultades, necesidades de protocolos y formas de aprendizaje servicio. Conclusión: Se encontró que el conocimiento, la práctica, la responsabilidad, la habilidad y la comunicación son factores que posibilitan el cuidado seguro de los niños y sus familias en situaciones de urgencia y emergencia.

Descriptores: Enfermería; Niños; Grupo de Enfermería; Medicina de Emergência Pediátrica.

\section{INTRODUÇÃO}

No âmbito pediátrico, surge uma crescente preocupação por parte dos serviços e dos profissionais de saúde, criar sistemas de cuidados para crianças que necessitam de atendimento de urgência. Este fato ocorre devido à rápida deterioração clínica do quadro clínico apresentado. ${ }^{1}$ Nesse sentido, têm sido adotadas estratégias efetivas de alerta precoce, desenvolvidas em contextos (inter)nacionais, com o intuito de auxiliar no reconhecimento do estado crítico da criança e desencadear o suporte necessário para sua recuperação. ${ }^{2,3}$

Os serviços de urgência destinado às crianças gravemente enfermas, são utilizados também por pacientes pediátricos com doenças mais simples e de tratamento ambulatorial, este fato provoca períodos de superlotação, contribuindo para o aumento do tempo de espera, impactando diretamente no atendimento das crianças com quadros agudos $\mathrm{e}$ graves. ${ }^{4}$ Vale ressaltar que há situações especificas em que crianças atendidas em serviços de emergências não necessitam desse serviço, uma vez que certos agravos 
poderiam ser resolvidos em locais de menor complexidade, como em unidades da atenção básica. ${ }^{4,5}$ Outra peculiaridade referente ao atendimento de crianças em situação de emergências pediátricas é a presença de acompanhantes, sendo este um direito garantido na Constituição Federal e regulamentado por meio do Estatuto da Criança e do Adolescente (ECA), indicando que o atendimento deve estar voltado para ações assistenciais ofertadas às crianças bem como aos seus acompanhantes. ${ }^{6}$

Além disso, os serviços e os profissionais que prestam assistência à criança em emergência enfrentam também problemas relacionados à fragmentação do trabalho causados por conflitos e assimetrias de poder; falta de habilidade técnica, comunicação ineficiente entre os diferentes profissionais de saúde e acompanhantes, estrutura física inadequada, recursos de materiais inadequados que dificultam a manutenção da segurança relacionada à assistência. $^{7}$ Estes fatores interferem na prática e constituem barreiras que afetam diretamente na qualidade da assistência e de possíveis riscos relacionados à segurança do paciente. ${ }^{5,7,8}$

Diante de tais considerações, para o exercício das atividades laborais em emergências hospitalares, são requeridas competências específicas da equipe de enfermagem, tais como: pensar rápido, ter agilidade e capacidade de resolver os problemas emergentes, que exigem atitudes e habilidades para afastar os riscos de morte iminente, de forma a garantir a qualidade de assistência para as crianças e seus familiares. ${ }^{9,10}$ Desse modo, o cuidar no âmbito emergencial pediátrico, tem como base ideológica salvar vidas de forma humanizada, envolvendo a ausculta qualificada e processo de comunicação efetivo voltada ao binômio: criança e acompanhante. $^{8,9}$

Nesta perspectiva, o processo de trabalho de enfermagem no setor de emergência pediátrica é um desafio para a equipe, apesar dos avanços quanto à definição de políticas de saúde que estabelecem a reorganização da rede de atenção às urgências no Brasil, os serviços hospitalares continuam sendo a principal porta de entrada de crianças em situações de emergências. ${ }^{8-10}$ Gerando sobrecarga de trabalho a estes profissionais, desse modo este estudo objetivou descrever as percepções da equipe de enfermagem de um Pronto Socorro Infantil sobre o atendimento à criança em emergência.

\section{MÉTODO}

Trata-se de uma pesquisa de abordagem qualitativa e descritiva. A escolha pela pesquisa qualitativa é justificada pela possibilidade de 
interpretar relações e comportamentos, procurando compreender significados e percepções que as pessoas constroem acerca dos mesmos, assim, fornece subsídios para que o cuidado prestado seja eficaz e humanizado. ${ }^{11}$

O cenário de coleta de dados foi uma Unidade de Pronto Socorro Infantil (PSI) de um hospital de ensino, localizado no estado de Minas Gerais. A unidade é composta de três enfermarias e uma sala de emergência, com estruturas necessárias para a assistência à criança em situação de risco iminente. O quadro de trabalhadores é composto por alguns profissionais como: médicos, enfermeiros e técnicos de enfermagem, fisioterapeuta e fonoaudiólogo.

Foram considerados como critérios de inclusão para produzir a análise, profissionais com atuação mínima de um ano na unidade estudada. Quanto aos critérios de exclusão, foram considerados profissionais licenciados, em cargo de gerenciamento, em férias e/ou cedidos para outros serviços.

Para se chegar à amostra final, utilizou-se se a técnica de saturação teórica. Essa técnica é definida como a suspensão de inclusão de novos participantes, quando os dados obtidos começaram a apresentar repetição e redundância. Para a efetivação desses processos foram utilizadas as seguintes etapas: disponibilizar os registros de dados brutos; imergir em cada registro; compilar as análises individuais, reunir os temas para cada categoria; codificar os dados; determinar os temas e categorias de enunciados; verificar e visualizar a saturação das categorias emergidas. ${ }^{12}$ Constatou-se a saturação teórica após a oitava entrevista, porém, seguiu-se com a coleta de dados até a décima entrevista, no intuito de reforçar a saturação.

A coleta de dados ocorreu em duas etapas interdependentes: na primeira utilizou-se um roteiro semiestruturado que constava a identificação das participantes, incluindo idade, sexo, formação profissional, tempo de atuação profissional, vínculo empregatício na instituição e carga horária semanal de trabalho. Essa etapa foi realizada por uma das pesquisadoras previamente capacitada.

Para a segunda etapa foram realizadas entrevistas individuais, através de questões abertas: "Como é para você atender uma criança em emergência? Em quais situações você sente mais dificuldade de realizar o atendimento na criança em emergência? Como você aprendeu a realizar o atendimento na criança em emergência? Gostaria de falar mais alguma coisa?" As duas etapas foram realizadas entre os meses de setembro a dezembro de 2018, o local de coleta foi uma sala privativa da unidade estudada designada pela chefia do serviço. As entrevistas foram 
gravadas através de mídia digital, com duração média de 15 minutos cada, posteriormente foram transcritas na íntegra. Para preservar a identidade das participantes do estudo, optou-se por denominá-las utilizando-se a inicial "E", referente à entrevista, seguida por um número arábico, em correspondência à sequência de inclusão das mesmas na pesquisa (E1, E2... E10).

Os dados foram inseridos no programa Microsoft Word® e submetidos à verificação de conteúdo modalidade temática, definida por ser um conjunto de técnicas de análise de informações, provenientes das falas das participantes investigadas sobre determinado assunto, possibilitando centralizar as ideias e categorizá-las por temas, seguindo-se as três etapas pré-estabelecidas: pró-análise, exploração do material e categorização dos dados. ${ }^{13}$

Para a realização do estudo, foram respeitadas as diretrizes e normas regulamentadoras de pesquisa com seres humanos, presentes na Resolução 466/12do Conselho Nacional de Saúde. O projeto, com registro CAAE: 79030017.9.0000.5154, foi aprovado pelo Comitê de Ética em Pesquisa sob parecer $n .^{\circ}$ 2.384.968. Todas as participantes concederam sua participação na pesquisa mediante assinatura no Termo de Consentimento Livre e Esclarecido.

\section{RESULTADOS}

Participaram do estudo 10 profissionais de enfermagem, todos do sexo feminino, sendo duas enfermeiras e oito técnicas de enfermagem, com idade entre 34 a 55 anos, com média de 46,5 anos. O tempo de experiência profissional foi de 9 a 30 anos, com média de 17,4 anos. Das participantes, uma enfermeira possui especialização em Enfermagem Pediátrica.

Após a transcrição e análise das entrevistas, foi realizada a categorização das falas, revelando cinco categorias, a seguir.

\section{Percepções e significados sobre o conhecimento do atendimento}

Percebeu-se, pelos relatos das participantes, que para atender a criança em emergência é exigido terem conhecimento: [...] é uma atuação delicada que exige muito conhecimento e preparo emocional (E1)

Segundo as participantes o conhecimento, faz com que a equipe se sinta segura para o atendimento à criança em emergência:

[...] conhecimento te dá muita segurança para agir conforme a situação (E2)

Para os membros da equipe, o atendimento à criança em emergência produz situações de tensão e ansiedade, sendo o conhecimento uma habilidade necessária para esse evento:

[...] é uma grande adrenalina onde ficamos ansiosos para chegarmos à um resultado positivo (E4) 
[...] apreensiva, porém, ciente de fazer o melhor naquilo que escolhi (E6)

As participantes relatam, que a espera da criança em emergência produz expectativas que podem ser superadas quando se tem conhecimento e presteza, possibilitando um desfecho positivo:

[...] na chegada sempre fico com muitas expectativas, às vezes com medo de não saber como agir [...], porém quando elas chegam, todos se juntam em um único intuito: fazer o melhor que podemos, então me sinto útil e feliz por poder ajudar (E8)

\section{Percepções e significados sobre a}

\section{responsabilidade no atendimento}

Essa categoria representa o relato das participantes sobre a importância de ser responsável. Novamente registram a relevância do conhecimento para prestação da assistência:

[...] a gente desdobra para fazer o procedimento com eficácia e rapidez, depende do estado da criança [...] da gravidade. (E3)

Para as participantes a responsabilidade é uma habilidade requerida para um bom atendimento:

O Pronto Socorro é uma unidade que requer de nós profissionais muita responsabilidade, agilidade, calma, [...] sempre será um momento de tensão, mas em equipe, fazemos sempre o melhor [...], pois cada paciente e situação será diferente e única. (E7)

\section{Percepções e significados sobre as}

\section{dificuldades no atendimento}

Dentre as dificuldades referidas pelas participantes destaca-se a presença dos pais:
Quando os pais estão presentes (E5)

As participantes apontam como dificuldades para o atendimento:

Quando a sala de emergência está cheia e não tem espaço para prestar o atendimento (E10)

A maior dificuldade é quando não encontramos materiais necessários e também quando a equipe médica por grande volume do pessoal impede a chegada da enfermagem (E9)

Indicam que a insegurança e a falta de profissionalismo são fatores que dificultam o atendimento: Quando deparamos com profissional inseguro

Com tumulto e falta de profissionalismo (E9)

Dentre as dificuldades referidas pelas participantes destacam o estado da criança: [...] pude sentir que tive dificuldades em crianças desacordadas [...] entendo que não há mais o que fazer. (E10)

\section{Percepções e significados sobre a necessidade de protocolos}

As participantes consideram que os protocolos assistenciais para o atendimento a criança em emergência são necessários e propiciam um desfecho positivo:

Totalmente necessário [...] temos possibilidades e condições para a realização dos procedimentos com mesmas regras e treinamentos (E3)

[...] usando protocolos poderemos oferecer cuidados necessários para um atendimento com sucesso (E5)

Elas acreditam que os protocolos assistenciais para o atendimento a criança em emergência possibilitam que os profissionais envolvidos tenham uma comunicação uniforme: 
Sim. Forma de todos falarem a mesma língua (E7)

Apontam os protocolos como sendo um guia para os profissionais com pouca experiência na assistência à criança em emergência:

[...] as pessoas inexperientes nesse caso, poderiam ter mais segurança nos procedimentos (E4)

\section{Percepções e significados sobre as formas} de aprendizagem do atendimento

As participantes destacam que a relação entre a teoria e prática voltadas à assistência da criança em emergência propiciam a aprendizagem:

Aprendi em cursos teóricos e na prática, principalmente no SAMU, e treinamento prático toda Sexta-feira, e também prestando o atendimento na sala de emergência. Aprendi na prática, na faculdade [...] (E6)

O ensino proposto pela instituição é um fator que contribui para o aprendizado e experiências práticas:

Aprendi com experiência no setor e treinamentos na instituição (E2)

Levantaram a importância dos estudos específicos na área de emergências pediátricas como contribuinte para o aprendizado:

[...] aprendi estudando sobre situação de emergência em pediatria (E5)

[...] aprendi na faculdade e no PALS (E8)

[...] aprendi com curso de urgência e emergência (E10)

Consideram a comunicação da equipe fundamental para o aprendizado da prática assistencial à criança em emergência:

A comunicação da equipe é fundamenta no aprendizado (E5)

Dentre os fatores contribuintes com o aprendizado da assistência à criança em emergência, as participantes destacam o auxílio da chefia e demais membros da equipe:

Aprendi através de orientações da minha chefia e colegas de trabalho (E3)

\section{DISCUSSÃO}

Com as informações obtidas, buscouse lançar um olhar sobre as percepções da equipe de enfermagem quanto à responsabilidade, a habilidade e a comunicação como fatores primordiais no atendimento às emergências pediátricas. Os profissionais entrevistados consideram que estes fatores possibilitam uma assistência segura, eficaz e com maiores oportunidades de chegar a um desfecho positivo. Estudos sobre essa temática mostram que os trabalhadores de enfermagem não se sentem preparados para lidar com situações críticas no cuidado à criança, assim como com a família neste cenário assistencial. 5,7,9,10,14

A percepção dos saberes e das práticas, no atendimento de emergência em unidade infantil foram evidenciadas nas falas das participantes como delicadas, que exigem da equipe conhecimento e preparo emocional para agir em acordo com a 
situação vivenciada. Estudos apontam que a equipe de enfermagem defronta com esforço físico, mental, emocional e psicológico, visto que a demanda de atenção para a realização desse processo de trabalho requer alto grau de complexidade, necessitando o preparo de um contexto receptivo para efetuar mudanças sustentáveis. ${ }^{9,10}$

As participantes entendem que a execução do trabalho desempenhado em uma unidade de emergência exige agilidade, iniciativa, habilidade para o trabalho em equipe, equilíbrio emocional e autocontrole em situações prioritárias. Corroborando com estudos sobre a temática. ${ }^{5,9,10-16}$

A assistência em setor de emergência pediátrica ocorre à inclusão da família/acompanhante, nesse sentido as participantes indicaram como dificuldade no atendimento quando os pais estão presentes. Estudos sobre essa questão apontam que essa prática não está incorporada nos serviços de saúde, embora o ECA normatize a presença dos pais e familiares. ${ }^{4,5,8,9}$. Esse tema tem sido frequentemente debatido ao nível mundial entre os profissionais da saúde, em particular a área de enfermagem, sugerindo que as equipes de saúde não estão familiarizadas com a presença da família/acompanhantes nos procedimentos de emergência. ${ }^{17,18}$ Embora alguns estudos demonstrem que equipes de enfermagem tem mais proximidade e diálogo com familiares das crianças hospitalizadas, assim como nos setores de urgência e emergência pediátrica. ${ }^{6-8,17,18}$

As participantes apontam que a dificuldade mais complexa é a condição de entrada da criança no atendimento de emergência, seja em relação à gravidade do quadro, ou em relação à questão emocional. Esta condição faz que essas profissionais sintam-se vulneráveis diante da situação vivenciada, implicando manifestações de emoções profundas, principalmente relacionadas à eminência da morte dessa criança. Vale registrar que a prática da enfermagem em serviços de emergência é confrontada continuamente com emoções negativas, relacionadas com o sofrimento das crianças e familiares..$^{9,10}$ Desse modo faz-se necessário que a equipe de enfermagem utilize evidências para obter melhora na qualidade da assistência, centralizando as ações não somente em si, mas também no cliente, buscando amenizar a situação em questão. ${ }^{17}$

As participantes destacaram a importância de ter protocolos assistenciais, para a padronização dos procedimentos, de maneira que todos envolvidos tenham ações em comum para uma assistência eficaz. A utilização de protocolos nos serviços de saúde está diretamente relacionada com a segurança do paciente. ${ }^{7,8,17}$ As ações 
educativas voltadas para os conteúdos dos protocolos, associando às práticas baseadas em evidências, contribuem para a mudança de padrões de prática. Este processo possibilita a melhoria da qualidade do cuidado. $^{7,19}$ Acrescido aos protocolos os registros padronizados contribuem para que a comunicação ocorra de forma eficaz e precisa. ${ }^{7,8,18,19}$

A atuação da equipe de enfermagem em serviços de urgência e emergência, demanda variados conhecimentos necessários à assistência de pacientes com necessidades complexas. Desse modo, romper os padrões já defasados e abrir novos meios de saber sobre determinado assunto trabalhado possibilita maior qualidade da assistência e também repercute na segurança do paciente, além de fortalecer o coletivo. ${ }^{7,19}$

\section{CONCLUSÃO}

Os resultados dessa pesquisa apontam que o conhecimento, prática, responsabilidade, habilidade, comunicação, equilíbrio emocional e autocontrole são fatores que possibilitam uma assistência segura à criança e seus familiares em situação de emergência, são habilidades primordiais para execução do trabalho nessas unidades.

As participantes indicaram que entre as dificuldades no atendimento, relacionase a presença dos pais e o estado da entrada da criança na emergência, seja em relação à gravidade do quadro, ou em relação à questão emocional, implicando manifestações de emoções profundas, principalmente relacionadas à eminência da morte dessa criança. Destacam a importância da existência de protocolos nas instituições, como fundamentais para o melhor desenvolvimento desse processo de trabalho, pois, com o profissional seguro a assistência tem maiores oportunidades de um desfecho favorável. A prática de educação continuada e permanente com frequência deve ser valorizada na totalidade e deve ser vista como investimento não somente no profissional, mas também na qualidade da assistência ao paciente.

Como fatores limitantes do estudo, considera-se a representação de um único cenário, ressaltando-se a necessidade da realização de novas pesquisas sobre esse assunto. As emergências pediátricas são pouco exploradas, mesmo com a riqueza de conhecimentos presentes nesta temática, proporcionando outros estudos a serem realizados.

\section{REFERÊNCIAS}

1 Amthauer C, Cunha Ml. Manchester Triage System: main flowcharts discriminators and outcomes of a pediatric emergency care. Rev LatinoAm Enfermagem [Internet]. 2016[cited 2018 May 13];12:e2779-85. Available from: http://www.scielo.br/pdf/rlae/v12/7.pdf 
2 Burokienè S, Raistenskis J, Burokaitė E, Čerkauskienè R, Usonis V. Factors Determining Parents' Decisions to Bring Their Children to the Pediatric Emergency Department for a Minor Illness. Med Sci Monit. 2017 Aug 28;23:4141-4148. doi:

10.12659/msm.902639. PMID: 28845042 ; PMCID: PMC5584823.

3 Macedo GPOS, D'Innocenzo M. Satisfação da qualidade de atendimento em um Pronto-Socorro Infantil. Acta paul. enferm. [Internet]. 2017 Dez [citado 2020 Nov 18]; 30( 6 ): 635-643. Disponível em:

http://www.scielo.br/scielo.php?script=s ci_arttext\&pid=S0103-

21002017000600635\&lng=pt. https://d oi.org/10.1590/1982-0194201700092

4 Neves FG, Moraes JRMM, Morais RCM, Souza TV, Ciuffo LL, Oliveira ICS. O trabalho da equipe em emergência pediátrica na perspectiva dos acompanhantes. Esc Anna Nery Rev Enferm. [Internet]. 2016 [citado em 21 maio 2018]; 20(3):e20160063.

Disponível em:

http://www.scielo.br/scielo.php?script=s ci_arttext\&pid=S1414-

81452016000300208. doi: 10.5935/1414-8145.20160063

5 Santos CKR, Moraes JRMM, Santos NLP, Souza TV, Morais RCM, Azevedo SD. Quality of nursing care in a pediatric emergency department: the companions' view. Rev Enferm UERJ [Internet]. 2016 [cited 2018 May 13];24(4):e17560. Available from: http://www.facenf.uerj.br/v24n4/ v24n4a03.pdf

6 Centro de Defesa dos Direitos da Criança e do Adolescente (Rio de Janeiro). ECA 2019 Estauto da Criança e do Adolescente [Internet]. Rio de Janeiro: CEDECA; 2019 [citado em 21 maio 2019]. p. 24-78. Disponível em: https://cedecarj.files.wordpress.com/201 9/06/livro-eca-2019-versc3a3o-internet1.pdf

7 Siman AG, Brito MJM. Mudanças na prática de enfermagem para melhorar a segurança do paciente. Rev. Gaúcha Enferm. [Internet]. 2016 [citado em 21 maio 2018]; 37:1-9. Disponível em: http://www.scielo.br/pdf/rgenf/v37nspe/ 0102-6933-rgenf-198314472016esp68271.pdf

8 Buboltz FL, Silveira A, Neves ET, Silva JH, Carvalho JS, Zamberlan KC. Percepção de familiares sobre sua presença ou não em situação de emergência pediátrica. Texto \& Contexto Enferm. [Internet]. 2016 [citado em 10 abr 2019]; 25(3):e0230015. Disponível em: http://www.scielo.br/pdf/tce/v25n3/pt_0 104-0707-tce-25-03-0230015.pdf. doi:http://dx.doi.org/10.1590/010407072016000230015

9 Lamb FA, Beck CLC, Coelho APF, Vasconcelos RO. Trabalho de enfermagem em pronto socorro pediátrico: entre o prazer e $\mathrm{o}$ sofrimento. Cogitare Enferm. [Internet]. 2019 [citado em 05 jun 2019]; 24:e59396. Disponível em: https://revistas.ufpr.br/cogitare/article/vi ew/59396. doi:dx.doi.org/10.5380/ce.v24i0.59396

10 Duarte MLC, Glanzner CH, Pereira LP. $\mathrm{O}$ trabalho em emergência hospitalar: sofrimento e estratégias defensivas dos enfermeiros. Rev Gaúcha Enferm. [Internet]. 2018 [citado em 05 jun 2019]; 39:e2017-0255. Disponível em:

http://www.scielo.br/scielo.php?script=s ci_arttext\&pid=S1983$14472018000100444 \& \operatorname{lng}=$ en. doi:https://doi.org/10.1590/19831447.2018.2017-0255

11 Polit DF, Beck CT, Hungler BP. Fundamentos de pesquisa em enfermagem: métodos, avaliação e utilização. $9^{a}$ ed. Porto Alegre: ArtMed; 2018.

12. Minayo MCS. Amostragem e saturação em pesquisa qualitativa: consensos e controvérsias. Rev Pesq Qualit. 2017 [citado 2010 nov 
16];5(7):1-12. Disponível em:

https://edisciplinas.usp.br/pluginfile.php

/4111455/mod_resource/content/1/

Minayosaturacao.pdf

13 Minayo, M. C. S. O desafio do conhecimento: Pesquisa qualitativa em saúde. São Paulo: Hucitec, 2013.

14 Macedo GPOS, D’Innocenzo M. Family members' satisfaction with the care flow in the Pediatric Emergency Department. Rev Bras Enferm [Internet]. 2019;72(2):435-41. doi: http://dx.doi.org/10.1590/0034-71672018-038

15 Oliveira JTO, Ermida PMV, Copelli FHS, Santos JLG, Erdmann AL, Andrade SR. Gerência do cuidado de enfermagem em unidades de pronto atendimento. Invest Educ Enferm. [Internet]. 2015 [citado em 10 abr 2019]; 33(3):406-14. Disponível em: http://www.scielo.org.co/scielo.php?scri $\mathrm{pt}=$ sci_arttext\&pid=S012053072015000300003

16 Silva JH, Buboltz FL, Silveira A, Eliane TN, Portela JL, Jantsch LB. Permanência de familiares no atendimento de emergência pediátrica: percepções da equipe de saúde. Rev Baiana Enferm. [Internet]. 2017 [citado em 21 jun 2019]; 31(3):e17427.

Disponível em:

https://portalseer.ufba.br/index.php/enfe rmagem/article/view/17427/0. doi:http://dx.doi.org/10.18471/rbe.v31i3 .17427

17 Grahn M.Olsson E.Mansson M.Interactions between children and pediatric nurses at the emergency department: A Swedish interview study.Journal of Pediatric

Nursing. 2016; 31: 284292https://doi.org/10.1016/j.pedn.2015. $\underline{11.016}$

18 Janhunen K,; Kankkunen P, Kvist T. Nursing Staff's Perceptions of Quality of Care for Children in Emergency Departments-High Respect, Low Resources.J Pediatr Nurs; 37: e10-e15, 2017.
DOI: https://doi.org/10.1016/j.pedn.201 7.08.029

19 Silva, J A et al. Índice de Gravidade de Emergência: acurácia na classificação de risco. Einstein (São Paulo) [online]. 2017, vol.15, n.4, pp.421-427. ISSN $2317-$

6385. http://dx.doi.org/10.1590/s167945082017 ao3964.

20 Paixão DPSS, Batista J, Maziero ECS, Alpenre FT, Amaya MR, Cruz EDA.

Adesão aos protocolos de segurança do paciente em emergência unidades de cuidados. Rev Bras Enferm. [Internet]. 2018 [citado em 21 maio 2018]; 71(Suppl 1):577-84. Questão Temática: contribuições e desafios da enfermagem práticas em saúde coletiva. Disponível em:

http://www.scielo.br/pdf/reben/v71s1/pt 0034-7167-reben-71-s1-0577.pdf. doi: http://dx.doi.org/10.1590/0034-71672017-0504

RECEBIDO: 01/12/2019

APROVADO: 08/02/2021

PUBLICADO: 06/2021 